\title{
Unlocking the Potential of
}

HIGH ENAROPY ALLOYS

with New TMS Study

Dan Miracle

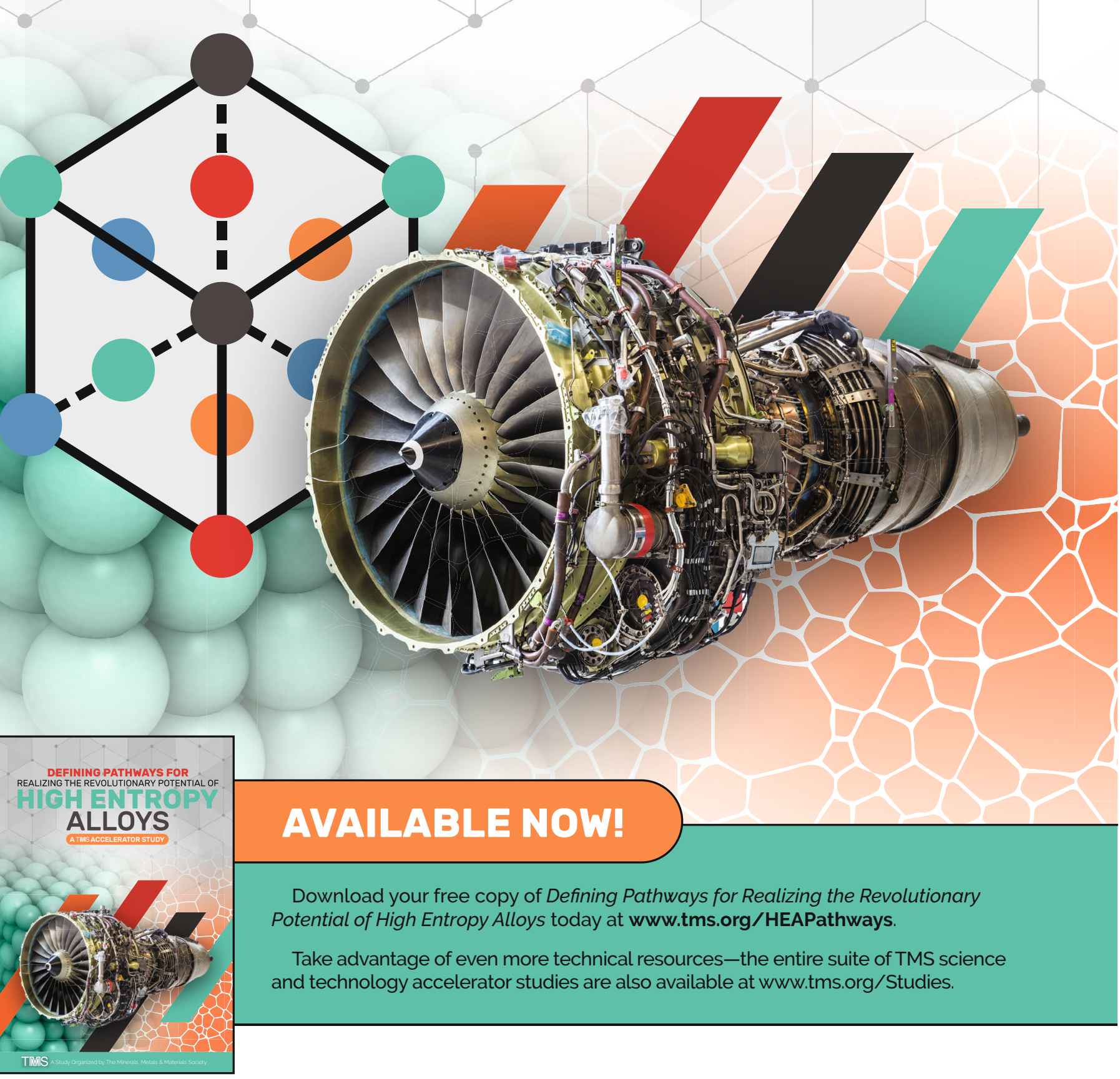




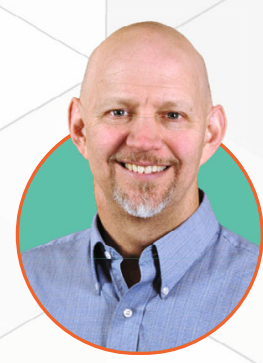

Dan Miracle
Defining Pathways for Realizing the Revolutionary Potential of High Entropy Alloys is the newest in the series of TMS accelerator studies. High entropy alloys (HEAs), also called complex, concentrated alloys (CCAs) or multi-principle element alloys (MPEAs), give a new approach to design and develop materials by mixing concentrated blends of three or more principle elements. They offer a vast range of new directions for basic research and applied development on essentially any class of inorganic material. Major new opportunities exist to overcome longstanding challenges in energy, defense, transportation, health, and environmental sectors. High entropy alloys offer a triple challenge due to the vast range of new compositions, a spectrum of new research topics, and a wide range of potential uses.

\section{"Major new opportunities exist to overcome longstanding challenges in energy, defense, transportation, health, and environmental sectors."}

The purpose of this TMS accelerator study is to develop a set of recommendations to help the technical and funding communities navigate the maze of HEA needs and opportunities. Sponsored by the Defense Advanced Research Projects Agency (DARPA) with the Air Force Research Laboratory (AFRL), this report emphasizes defense applications, but many other applications are also included. The study team consisted of 14 internationally recognized subject matter experts from academia, industry, and government who volunteered significant time to this project. A product of the COVID-19 environment, this report was produced by convening these experts in several online meetings, teleconferences, and virtual facilitated workshops. The study team provided content, and the writing, editing, and reviewing of the report was done by both TMS staff and the study team. A separate team of nine international experts provided an independent review of the report prior to publication.

After a brief introduction to the current state of HEA technologies, Defining Pathways: (1) gives a prioritized list of applications and alloy domains of most promise, particularly for defense-related applications; (2) identifies the key technology gaps, barriers, and enablers of the next stage of research in areas with the greatest potential for near term, substantive impact; and (3) provides recommendations and action plans to fuel significant progress within the next three to five years in the scientific exploration, engineering development, and industrial implementation of HEAs. A quick overview of the top-level findings described in the report are highlighted in this article.
A total of 16 applications and alloy classes were identified as high priority (see Table I). The first four are all materials for extreme temperature environments, and the highest priority is for refractory HEAs (RHEAs). These RHEAs provide new approaches to achieving many longstanding, high-impact aspirations in defense, energy, and transportation sectors. High entropy ceramic materials are included in the list of extreme temperature materials, as are thermal barrier coatings and, at the other extreme of the temperature spectrum, cryogenic materials. HEAs for catalysts and electrochemical uses, such as batteries and practical theromoelectric materials, offer high priority opportunities, and a range of applications that require resistance to environmental degradation, including corrosion, oxidation, marine fouling, and bacterial growth are also included. The ability to replace expensive or hard-to-get elements such as platinum or rare earth metals via concentrated alloying with multiple elements is a priority, and HEAs to enable alternative fuels, including hydrogen storage materials, are also on the list. High entropy brasses and bronzes, with applications in defense, health, and environmental industries, are included as the most likely HEAs to reach first application. It is perhaps a strange twist of fate that alloys produced by humanity's first venture into alloyed metals, brasses and bronzes, may also be the first to be improved by the high entropy concept.

Table I. List of proposed target material classes and applications for HEA insertion:

1. Refractory HEAs (RHEAs)

2. Ultrahigh-temperature high entropy ceramics (HECs)

3. Thermal barrier coatings (TBCs)

4. Cryogenic systems

5. Catalysts

6. Corrosion-resistant coatings

7. Light-weight materials

8. Novelty HEA systems including:

a. Replacements for rare-earth and critical materials systems

b. Bulk metallic glasses with corrosion resistant properties

c. Anti-fouling or anti-bacterial coatings

d. Encapsulating frameworks for storing gases, reactants, or corrosion inhibitors

9. Batteries/superconductors

10. Thermoelectric materials

11. Electromagnetic application HEAs

12. HEAs with anti-bacterial properties/ applications

13. HEAs to enable renewable/alternative fuels

14. Quantum computing materials

15. Hydrogen-compatible/storage materials

16. High-entropy brasses and bronzes

Table I. This table presents potential application areas for high entropy alloys as defined in the Defining Pathways report. (Defining Pathways for Realizing the Revolutionary Potential of High Entropy Alloys, 2021, p. 14.) 
Nine key challenge areas are identified and described in the report (see Table II). High throughput screening methods are essential to explore the vast, new composition space (HEAs expand the number of new alloy bases by up to a factor of $10^{7}$ ) and therefore are at the top of the list. The combination of three or more concentrated elements requires the development of new scientific theories, predictive models, and computational tools, and is listed next. Other challenges include availability of high throughput, high temperature test equipment, reliable sources of high-quality material feedstock (especially powder), expanded thermodynamic databases, and development of new composition-structureprocessing-properties relationships. Though it appears at the end of the list, the need to train the next generation workforce in these new theories and methods is a challenge with particularly high importance.

\begin{tabular}{|c|c|}
\hline Key Challenge Area & Needs/Current Limitations \\
\hline $\begin{array}{l}\text { A. High-Throughput Screening } \\
\text { Methods and Experimental Tools }\end{array}$ & $\begin{array}{l}\text { - High-throughput, automated, and/or autonomous tools and processes for } \\
\text { integrated synthesis, characterization, and evaluation of HEAs } \\
\text { - High-throughput experimental approaches for melting temperature; } \\
\text { tensile strength and ductility; and toughness } \\
\text { - High-throughput surrogate experiments for expensive and/or slow tests }\end{array}$ \\
\hline $\begin{array}{l}\text { B. Predictive Models and } \\
\text { Computational Tools }\end{array}$ & $\begin{array}{l}\text { - Fundamental theory for complex compositional space } \\
\text { - Uncertainty-based predictive computational models for HEA } \\
\text { development } \\
\text { - Accurate cross-potentials for computational models } \\
\text { - Computational tools for predicting structural and functional properties } \\
\text { - ML approaches to help guide alloy selection } \\
\text { - Visualization tools for interpreting complex phase spaces }\end{array}$ \\
\hline $\begin{array}{l}\text { C. High-Temperature Equipment } \\
\text { and Testing }\end{array}$ & $\begin{array}{l}\text { - High-temperature processing, testing, and property measurements } \\
\text { - Addressing simultaneously the constraints of processing conditions } \\
\text { (heating and oxidation) and sample size for high-temperature testing } \\
\left.\text { - Methods to process high melting point (e.g., }>2000^{\circ} \mathrm{C}\right) \text { RHEAs } \\
\text { - Robust high-temperature die materials }\end{array}$ \\
\hline $\begin{array}{l}\text { D. Scattered Data with Uncertain } \\
\text { Materials Pedigree }\end{array}$ & $\begin{array}{l}\text { - Robust, coordinated, pedigreed datasets to supplant the disparate current } \\
\text { data across the wide spectrum of HEA compositions } \\
\text { - Widely adopted schema to establish provenance for HEA metadata }\end{array}$ \\
\hline $\begin{array}{l}\text { E. Fundamental Composition- } \\
\text { Processing-Microstructure- } \\
\text { Properties Knowledge }\end{array}$ & $\begin{array}{l}\text { - Enhanced composition-processing-microstructure-properties correlations } \\
\text { with as broad an applicability range as possible }\end{array}$ \\
\hline F. In Situ Characterization Methods & $\begin{array}{l}\text { - In situ monitoring and characterization tools to track all test parameters } \\
\text { - Ability to monitor microstructural evolution in situ }\end{array}$ \\
\hline G. Thermodynamic Databases & $\begin{array}{l}\text { - Publicly available thermodynamic databases for HEAs } \\
\text { - Consistency across methods used to gather HEA data } \\
\text { - Multicomponent data to extrapolate into un-explored space } \\
\text { - Entropy properties (in thermodynamic databases) that are efficient and } \\
\text { flexible }\end{array}$ \\
\hline H. Availability of Affordable Powder & - Solutions to overcome prohibitively expensive HEA raw material costs \\
\hline $\begin{array}{l}\text { I. Workforce Trained in HEA } \\
\text { Exploration and/or Development }\end{array}$ & $\begin{array}{l}\text { - A workforce skilled in using experimental and/or computational } \\
\text { approaches and tools geared toward HEA exploration and development }\end{array}$ \\
\hline
\end{tabular}

Table II. This table presents high-priority challenge areas for future R\&D of HEAs on the left, while also cataloguing specific needs and/or limitations in each of these areas on the right. (Defining Pathways for Realizing the Revolutionary Potential of High Entropy Alloys, 2021, p. xxvi.) 


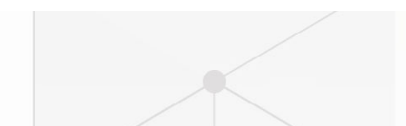

These challenges are used to form 36 specific, actionable recommendations that can guide future research and development efforts. Some of these recommendations also address other aspects of the science and technology enterprise-efforts to coordinate government agency funding, to form consortia that share pre-competitive data, and to conduct student-oriented, multi-institute HEA workshops and programs are listed, for example. Finally, the challenges and recommendations are combined to produce five coherent action plans. Each action plan consists of detailed tasks to more fully describe the needed efforts. For example, one of the tasks in the action plan for high throughput evaluation and testing moves beyond conventional high throughput methods by describing an autonomous materials research capability tailored for the challenges introduced by HEAs (see Figure 1). The action plans also give the recommended duration, progress metrics, estimated costs, and the types of key players and roles required for success. The action plans in Defining Pathways give stakeholders a detailed framework of prioritized recommendations to help accelerate the discovery, development, and implementation of these potentially game-changing materials.
"The action plans in Defining Pathways give stakeholders a detailed framework of prioritized recommendations to help accelerate the discovery, development, and implementation of these potentially game-changing materials."

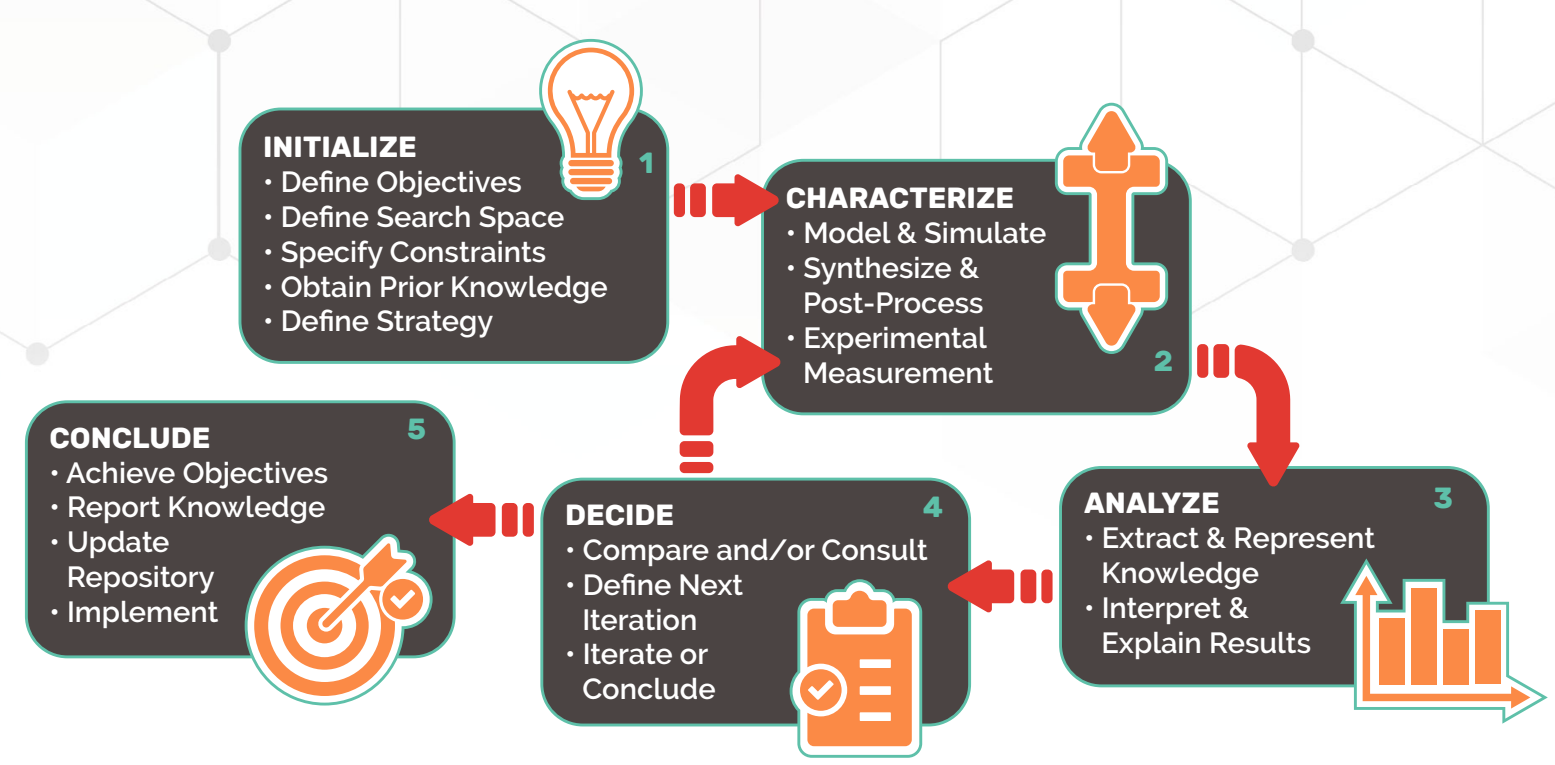

Figure 1. This figure represents the autonomous materials research (AMR) process, which incudes artificial intelligence (Al) and unsupervised machine learning (ML). (Defining Pathways for Realizing the Revolutionary Potential of High Entropy Alloys, 2021, p. 42.)

Dan Miracle is a Senior Scientist in the Materials and Manufacturing Directorate of the Air Force Research Laboratory (AFRL). He is the Defining Pathways study team lead and current chair of the TMS Structural Materials Division. 